\title{
THE INTERVAL ANALYTIC HIERARCHY PROCESS: SIMULATION EXPERIMENTS $^{1}$
}

\author{
Josef Jablonsky \\ Department of Econometrics, University of Economics \\ Praha, 13067 Czech Republic \\ jablon@vse.cz,www: http://nb.vse.cz/ jablon/
}

\begin{abstract}
The paper is concerned with the analysis and the utilisation of the simulation techniques in solving multicriteria decision making problems and especially discusses possibility of application of simulation approaches in the Interval Analytic Hierarchy Approach (IAHP). There are described simulation experiments with the IAHP by using different probabilistic distributions and compared given results. The simple procedure for ranking of alternatives is developed. The computational experiments are discussed with respect to the time consumption and other factors.
\end{abstract}

\section{Introduction}

Simulation modelling belongs to one of the often-used operational research techniques in the economic practice. It makes possible complete global analysis even of very complex decision making problems. Simulation techniques often represent the only possible way for solving some classes of operational research problems. The popularity of simulation approaches results from their simplicity and possibility to present given results in the nature and close way to decision makers. Nevertheless their big disadvantage consists in the fact that the simulation techniques are usually very computational demanding and time-consuming and require usually a specialised simulation software.

This paper is concerned with the analysis and the utilisation of the simulation techniques in multicriteria decision making especially we discuss possibility of application of simulation approaches in the Interval Analytic Hierarchy Process (IAHP).

\section{The Interval Analytic Hierarchy Process}

The Analytic Hierarchy Process (AHP) represents a powerful tool in analysis of the multicriteria decision problems with the finite set of alternatives. Regarding to the current development of the AHP we can summarise only its main features in this section. The full exposition is given e.g. in Saaty (1990).

The AHP starts from the fact that decision problems can mostly be organised into a hierarchy. The hierarchy represented a decision problem contains always several levels. The first (topmost) level defines a main goal of the decision problem and the last (lowest) level describes usually the decision alternatives. The levels between the first and the last level can contain secondary goals, criteria and subcriteria of the decision problem. The number of the levels is not limited, but in the typical case it does not exceed four or five. In this paper we consider only the simple three-level hierarchy which can represent the classical

1 The paper is supported by the Grant Agency of Czech Republic - grant no. 402/98/1488 and corresponds to the research programme of the Faculty Informatics and Statistics of the University of Economics Prague no. CEZ: J18/98:311401001. 
decision problem with the finite set of alternatives - evaluation of n-alternatives $X_{1}, X_{2}, \ldots, X_{n}$, by kcriteria $Y_{1}, Y_{2}, \ldots, Y_{k}$, (Fig. 1).

The principle of the AHP consists in pairwise comparisons of the elements in the separate hierarchy levels. The decision maker has to express his preferences and determine relation between the pairs of elements in the present level with respect to the element of the preceding level during the comparison. The information following from decision maker judgements in the given level of the hierarchy is synthesised onto the local priorities. They can express, e.g. relative importance of criteria (weight coefficients - in Fig. 1 denoted by $\left.v_{j}, i=1,2, \ldots, k\right)$ or preference indices of the alternatives with respect to the given criterion $\left(w_{i j}, i=1,2 \ldots, n, j=1,2, \ldots, k\right)$. Finally, the global priorities for all alternatives $u\left(X_{i}\right)$, $\mathrm{i}=1,2, \ldots, \mathrm{n}$, are found by synthesising the local priorities. In our simple case

$$
u\left(X_{i}\right)=\sum_{j=1}^{k} v_{j} w_{i j}, \quad i=1,2, \ldots, n .
$$

Then, the global priorities define the final preference order of the alternatives.

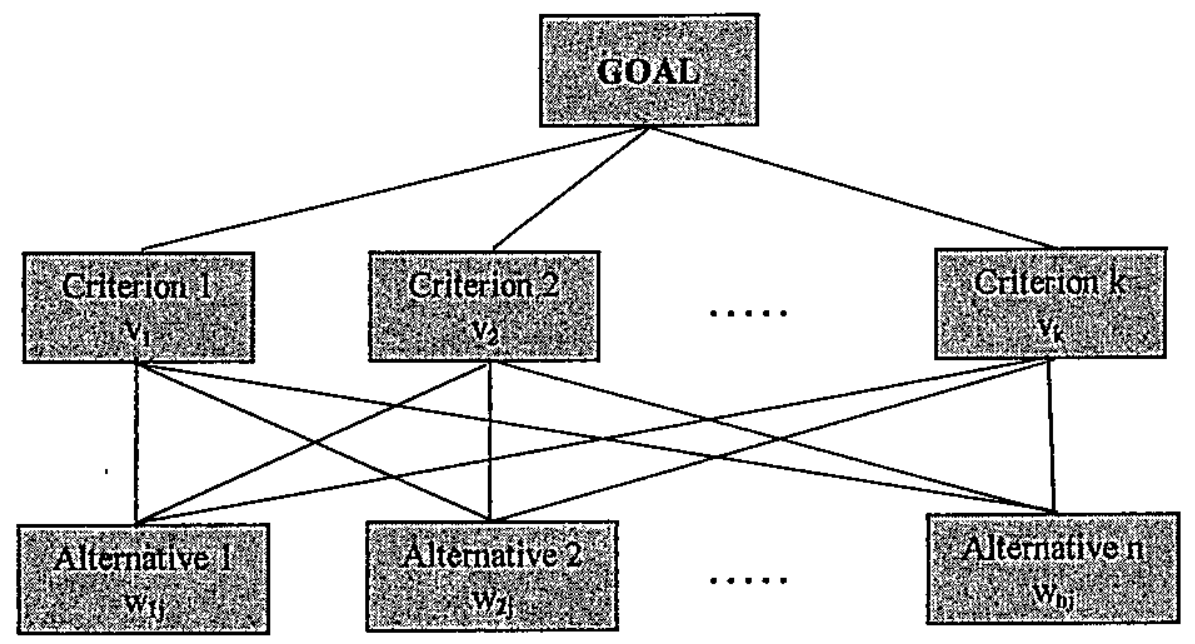

Fig. 1: Three-level hierarchy.

In the standard AHP model the decision maker judgements are organised into pairwise comparison matrices at each level of the hierarchy. The judgements are point estimates of the preference between two elements of the level. Let us denote the pairwise comparison matrix $A=\left\{a_{i j} \mid a_{j i}=1 / a_{i j}, a_{i j}>0, i, j=1,2, \ldots, k\right\}$, where $k$ is the number of elements of the particular level. Saaty proposes to use for preference expression $a_{i j}$ integers in the range 1 through 9 only, where 1 means that the $i$-th and the $j$-th element are equally important and 9 means that the $i$-th element is absolutely more important than the j-th element. The local priorities are derived by solving the eigenvector problem:

$$
\begin{aligned}
& \text { A.v }=\lambda_{\max } \mathrm{v}, \\
& \sum_{\mathbf{i}=1}^{\mathrm{k}} \mathrm{v}_{\mathrm{i}}=1,
\end{aligned}
$$

where $\lambda_{\max }$ is the largest eigenvalue of $A$ and $v$ is the normalised right eigenvector belonging to $\lambda_{\max }$.

In the deterministic AHP approach the decision maker always specifies point estimates that express his preference relations between two elements in the given hierarchy level. It can often be very strong and difficult to fulfil this condition for decision makers. They feel much better and closer to have the possibility to express their preference as interval estimates. For instance, instead of giving that the $\mathrm{i}$-th element is four times as preferable as the $j$-th element, he can assert that the $i$-th element is at least two but no more than five times as preferable as the $\mathrm{j}$-th element. 
The AHP model with interval decision maker judgements is usually called interval AHP (IAHP) model. It is characterised by interval comparison matrices given as follows:

$$
\mathrm{A}=\left[\begin{array}{cccc}
1 & \left\langle\mathrm{p}_{12}, \mathrm{q}_{12}>\right. & \cdots & <\mathrm{p}_{1 \mathrm{k}}, \mathrm{q}_{1 \mathrm{k}}> \\
\left\langle\mathrm{p}_{21}, \mathrm{q}_{21}\right\rangle & 1 & \cdots & <\mathrm{p}_{2 \mathrm{k}}, \mathrm{q}_{2 \mathrm{k}}> \\
\vdots & \vdots & \cdots & \vdots \\
\left\langle\mathrm{p}_{\mathrm{k} 1}, \mathrm{q}_{\mathrm{k} 1}\right\rangle & \left\langle\mathrm{p}_{\mathrm{k} 2}, \mathrm{q}_{\mathrm{k} 2}>\right. & \cdots & 1
\end{array}\right]
$$

where $p_{i j}$ is lower bound and $q_{i j}$ upper bound for preference relation $\left(a_{i j}\right)$ between the $i$-th and $j$-th element. Due to the reciprocal nature of the pairwise comparison matrices the relation $\mathrm{p}_{\mathrm{ij}} \cdot \mathrm{q}_{\mathrm{ji}}=1$ holds for all $\mathrm{i}, \mathrm{j}=1,2, \ldots, \mathrm{k}$.

The judgements in the IAHP can be considered as random variables defined over the given interval. In this way the IAHP changes from the deterministic model to the model with some stochastic features. That is why it cannot be analysed in the traditional way - by solving the eigenvector problem (1). It is necessary to look for new approaches that will respect interval inputs. The random variables for description of interval judgements can be selected from the available probabilistic distributions. We recommend to use either normal distribution with expected value $\mu_{i j}$ and standard deviation $\sigma_{i j}$ or general distribution with parameters $\mathrm{p}_{\mathrm{ij}} \leq \mathrm{c}_{\mathrm{ij}} \leq \mathrm{d}_{\mathrm{ij}} \leq \mathrm{q}_{\mathrm{ij}}$ that can be modified by setting of its parameters. The shapes of selected probability density functions are presented on Fig. 2.
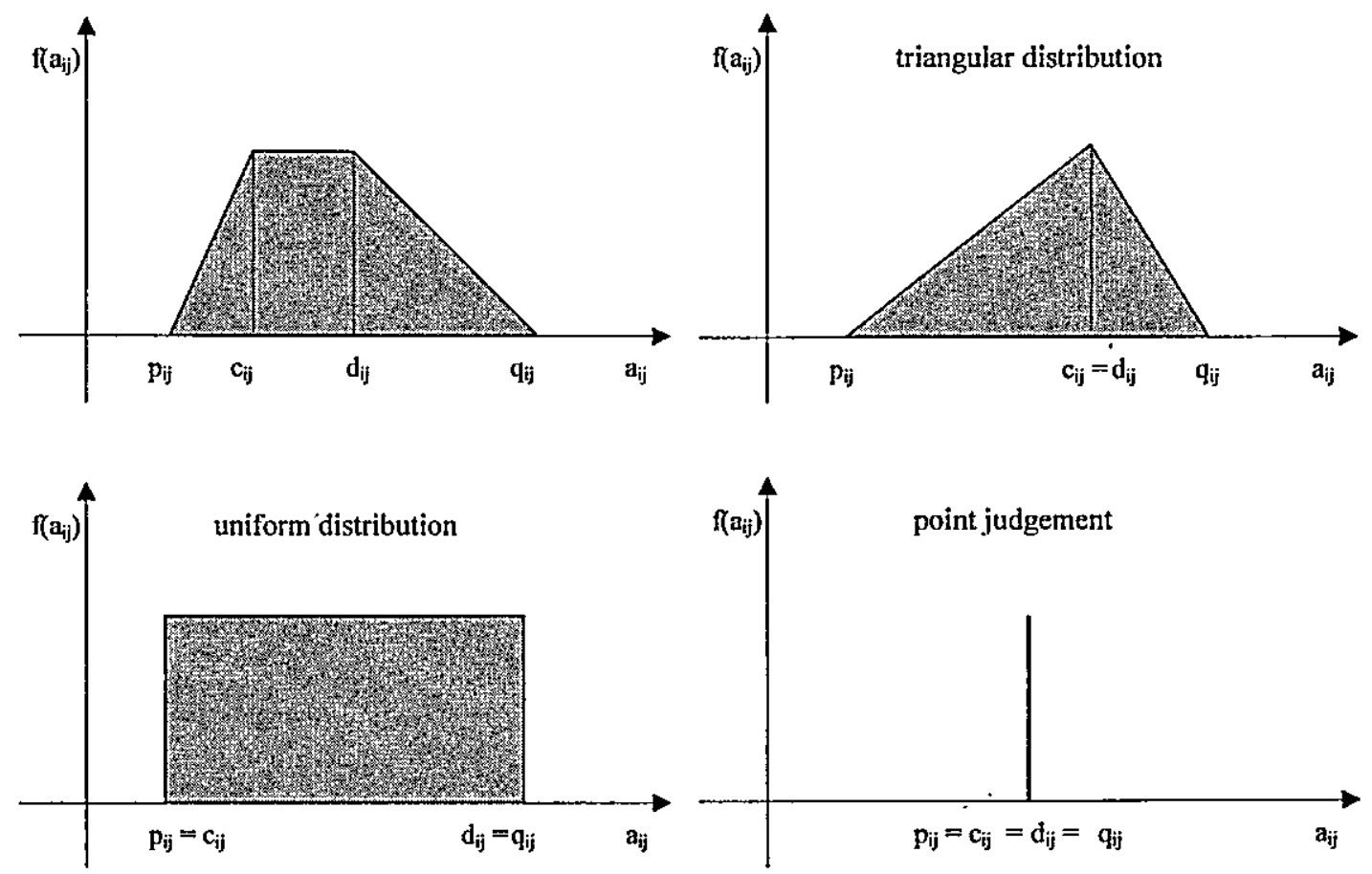

Fig. 2: Probability density functions of general distribution.

In the IAHP the question is how to derive the local priority coefficients from information contained in matrix (2). The traditional approach - solving the eigenvector problem (1) - is not acceptable due to the interval nature of the pairwise comparison matrix coefficients. It can be recognised two basic approaches discussing this problem. The first one is based on transforming the given problem. This transformation consists in solving series of typical single criteria linear programming problems - intervals for local and finally for global priorities are derived in this approach - e.g. see Arbel (1989) and Salo (1990). The second method is a simulation approach that derives local and global priorities by repeating a number of 
simulation steps - e.g. see Jablonsky (1991) and Saaty,Vargas (1987). One of the possible simulation approaches is presented and discussed in the next section of this paper.

\section{Simulation analysis of the IAHP}

Let us suppose that the $a_{i j}$ elements of pairwise comparison matrices $A$ are random variables $A_{i j}$ characterised by probability density function $f\left(a_{i j}\right)$ and cumulative probability function $F\left(a_{i j}\right)$. Let us consider the following probability distributions for these random variables:

- general distribution with probability density function defined by a linear broken function with $\mathrm{p}_{\mathrm{i} j} \leq$ $\mathrm{c}_{\mathrm{ij}} \leq \mathrm{d}_{\mathrm{ij}} \leq \mathrm{q}_{\mathrm{ij}}$ parameters (Fig. 2) and

- normal distribution with expected value $\mu_{\mathrm{ij}}$ and standard deviation $\sigma_{\mathrm{ij}}$.

Fig. 2 shows that the uniform distribution is only a special case of the general distribution for $p_{i j}=c_{i j}$ and $q_{i j}=d_{i j}$. By setting $p_{i j}=c_{i j}=d_{i j}=q_{i j}$ we can get a point judgements typical for standard AHP models. Because of the fact that we know the function model of the probability density function (it follows clearly from Fig. 2) we can derive the function model of the cumulative distribution function in this simple case. By inverse transformation

$$
F\left(a_{i j}\right)=r \Rightarrow a_{i j}=F^{-1}(r),
$$

where $r$ is a random number (a value of the random variable with uniform distribution over $<0,1$ )), we can get the function model (3) for generating values of the random variable Aij:

$$
\begin{array}{ll}
a_{i j}=p_{i j}+\sqrt{r\left(c_{i j}-p_{i j}\right) K}, & r \in<0, \frac{c_{i j}-p_{i j}}{q_{i j}-p_{i j}}>, \\
a_{i j}=\left(r K+p_{i j}+c_{i j}\right) / 2, & r \in<\frac{c_{i j}-p_{i j}}{q_{i j}-p_{i j}}, \frac{d_{i j}-p_{i j}}{q_{i j}-p_{i j}}>, \\
a_{i j}=b_{i j}-\sqrt{b_{i j}^{2}+\left(d_{i j}-q_{i j}\right)\left(r K+c_{i j}+p_{i j}\right)-d_{i j}^{2}}, & r \in<\frac{d_{i j}-p_{i j}}{q_{i j}-p_{i j}}, 1>.
\end{array}
$$

where $K=\left(q_{i j}-p_{i j}\right)+\left(d_{i j}-c_{i j}\right)$. If we set $p_{i j}=c_{i j}$ and $d_{i j}=q_{i j}$ we can get the simple function model for generation of values of the uniform distribution over the interval $<p_{i j}, q_{i j}$ ):

$$
\left.a_{i j}=r K / 2+p_{i j}, \quad r \in<0,1\right) .
$$

The values of the normal distribution were generated by means the well known Box-Muller transformation:

$$
\begin{aligned}
& a_{i j}=\mu_{i j}+\sigma_{i j} \sqrt{-2 \ln r_{1}} \sin \left(2 \pi r_{2}\right), \\
& a_{i j}=\mu_{i j}+\sigma_{i j} \sqrt{-2 \ln r_{1}} \cos \left(2 \pi r_{2}\right)
\end{aligned}
$$

where $r_{1}, r_{2}$ is the pair of random numbers.

The simulation analysis of the interval AHP model can proceed in several steps. They are given in brief as follows:

1. Specification of the probabilistic distribution for all the possible pairs of elements in the particular level of the hierarchy. If the normal distribution is used the parameters $\mu_{i j}$ and $\sigma_{i j}$ must be set. The $p_{i j}$, $c_{i j}, d_{i j}, q_{i j}$ parameters must be determined if the general distribution is preferred.

2. In $\mathrm{N}$ simulation steps:

- pairwise comparison matrices are generated,

- relative importance coefficients of criteria $v_{j}(j=1,2, \ldots, k)$ and preference indices of alternatives with respect to the $j$-th criterion $w_{i j}(i=1,2, \ldots, n ; j=1,2, \ldots k)$ are derived by solving $(1)$ or by one of the approximate methods - see Saaty (1990), 
- finally global priorities $\mathrm{u}\left(\mathrm{X}_{\mathfrak{i}}\right)(\mathrm{i}=1,2, \ldots, \mathrm{n})$ are computed; they define rank order of alternatives in the current simulation step.

3. The results given by $\mathrm{N}$ simulation steps can be organised into the rank order matrix $\mathbf{P}=\left\{p_{i j}\right\}$ $\mathrm{i}, \mathrm{j}=1,2, \ldots, \mathrm{n}\}$ :

\begin{tabular}{|c|cccc|c|}
\hline ranking & 1 & 2 & $\cdots$ & $\mathrm{n}$ & $\Sigma$ \\
\hline $\mathrm{X}_{1}$ & $\mathrm{p}_{11}$ & $\mathrm{p}_{12}$ & $\cdots$ & $\mathrm{p}_{1 \mathrm{n}}$ & $\mathrm{N}$ \\
$\mathrm{X}_{2}$ & $\mathrm{p}_{21}$ & $\mathrm{p}_{22}$ & $\cdots$ & $\mathrm{p}_{2 \mathrm{n}}$ & $\mathrm{N}$ \\
$\vdots$ & $\vdots$ & $\vdots$ & $\cdots$ & $\vdots$ & $\vdots$ \\
$\mathrm{X}_{\mathrm{n}}$ & $\mathrm{p}_{\mathrm{n} 1}$ & $\mathrm{p}_{\mathrm{n} 2}$ & $\cdots$ & $\mathrm{p}_{\mathrm{nn}}$ & $\mathrm{N}$ \\
\hline$\Sigma$ & $\mathrm{N}$ & $\mathrm{N}$ & $\cdots$ & $\mathrm{N}$ & \\
\hline
\end{tabular}

Elements of the rank order matrix express how many times the $\mathrm{i}$-th alternative is on the $\mathrm{j}$-th order in the global ranking during the simulation process and the ratio $\mathrm{p}_{\mathrm{i}} / \mathrm{N}$ can be interpreted as the rank order probability (probability that the $\mathrm{i}$-th alternative will be on the $\mathrm{j}$-th order in the final ranking).

4. Global rank order index is computed as follows

$$
\mathrm{g}\left(\mathrm{X}_{\mathrm{i}}\right)=\frac{1}{\mathrm{~N}} \sum_{\mathrm{j}=1}^{\mathrm{n}} \mathrm{j} \cdot \mathrm{p}_{\mathrm{ij}}, \quad \mathrm{i}=1,2, \ldots, \mathrm{n} .
$$

The index $g\left(X_{i}\right)$ can be interpreted as the average, rank order of alternative $X_{i}$. These indices define the final ranking of alternatives in this way.

In the AHP it is necessary to respect a consistency conditions of pairwise comparison matrices. The consistency of comparison matrices is measured by consistency index $\left(\lambda_{\max }-\mathrm{k}\right) /(\mathrm{k}-1)$. The value of this index is compared to the average consistency index of order $k$. The matrix consistency is considered to be satisfactory if the consistency ratio - consistency index divided by average consistency index - does not exceed 0.1 .

During the simulation it is necessary to check the consistency of generated matrices. Nevertheless, the computational experience show that the consistency of generated matrices is satisfactory if the following conditions are fulfilled:

- the consistency ratio of matrix $\left[E\left(a_{i j}\right)\right]$, where $E\left(a_{i j}\right)$ is the expected value of the random variable $A_{i j}$, is very good (the consistency ratio gets near to zero),

- the maximum recommended width of the range $\left\langle p_{i j}, q_{i j}\right\rangle$ is 3 if random variables $A_{i j}$ are defined over the range $<\mathrm{p}_{\mathrm{ij}}, \mathrm{q}_{\mathrm{ij}}>$.

The second condition is easy to respect - the recommended value $(q-p)<3$ is usually enough high for decision makers. The first condition must be checked during the specification of parameters of random variables. The simulation experience demonstrate that more than $95 \%$ of generated matrices are satisfactory with respect to their consistency if the conditions above are respected.

\section{Conclusions}

Simulation approach is one of the possible ways for analysing of complex decision problems. The results received by testing numerical examples show that the time of the simulation is acceptable for AHP models with maximum number of 7 elements in one hierarchy level. Table 1 demonstrates the time for 1000 simulation steps for different AHP models with three levels and the same number of alternatives and criteria - $\mathrm{k}=\mathrm{n}$. The experiments were realised on PC $586 / 350 \mathrm{MHz}$. The weights of the criteria and the preference indices for alternatives were computed in all cases by solving the eigenvector problem. By application one of the approximate methods (geometrical means of the elements in single rows of the pairwise comparison matrix) the results are given in significantly shorter time. The results given in Table 
2 show that the simulation procedure can be used (with respect to the time of the simulation) even for problems with the number of hierarchical levels greater than 3 .

\begin{tabular}{|l|c|c|c|c|c|}
\hline \multirow{3}{*}{ Distribution } & \multicolumn{5}{|c|}{ time of the simulation (sec) } \\
\cline { 2 - 6 } & $\mathrm{k}=7$ & $\mathrm{k}=6$ & $\mathrm{k}=5$ & $\mathrm{k}=4$ & $\mathrm{k}=3$ \\
\hline Uniform & 20 & 11 & 6 & 3 & 2 \\
\hline General & 24 & 14 & 8 & 3 & 2 \\
\hline Normal & 26 & 15 & 8 & 4 & 2 \\
\hline
\end{tabular}

Table 1: Simulation time.

\section{References}

Arbel, A. (1989), "Approximate Articulation of Preference and Priority Derivation", European Journal of Operational Research, 43, 317-326.

Jablonsky, J. (1991), "The Analytic Hierarchy Approach: A Simulation Approach", Proceedings of the International Workshop on MCDM, Prague, 68-78.

Saaty, T.L. (1990), The Analytic Hierarchy Process, RWS Publications, Pittsburgh.

Saaty, T.L.,Vargas, L.G. (1987), "Uncertainty and Rank Order in the AHP", European Journal of Operational Research, 32, 107-117.

Salo,A., Hamalainen,R. (1990), "Processing Interval Judgements in the Analytic Hierarchy Process", Proceedings of the $L X-$ th Conference on MCDM, Fairfax. 